\title{
The U.S. Department of Energy Plans to Close LANSCE \\ Roger Pynn
}

The following was originally written for Neutron News (Volume 4, No. 4) and is reprinted here with permission.

The attitude of society and political institutions toward research is changing. There is increasing demand that scientists solve problems which will benefit mankind on a relatively short time scale. Opposition to expensive projects such as the Superconducting Super Collider is increasing, and traditional nuclear physics has lost some of the support that it enjoyed for many decades. One might think that this "sea change" would favor neutron scattering-after all, this field has made and will continue to make important contributions to subjects such as materials science and structural biology that are enjoying an increase in popularity. Even more importantly, neutrons are being applied increasingly to problems of industrial interest.

There is at least one case in which the eroding support for nuclear physics is likely to have an adverse impact on neutron scattering. The U.S. Department of Energy (DOE) has announced its intention to close the Los Alamos Meson Physics Facility (LAMPF) in October of this year. This facility, which is funded primarily for nuclear physics research, also provides the proton beam that is used to generate neutrons at the Manuel Lujan Jr. Neutron Scattering Center (LANSCE). As a consequence, the current DOE plan will also result in the closure of LANSCE.

LANSCE is one of the world's most intense pulsed spallation neutron sources, with a peak flux that is comparable with that available at the world's leading spallation source, ISIS, in the United Kingdom. Starting in 1987, DOE invested about $\$ 20$ million in new buildings and spectrometers. LANSCE has operated for the past four years as a national user facility, open to researchers from universities, federal laboratories, and industry. A program advisory committee, shared with the Intense Pulsed Neutron Source at Argonne National Laboratory, judged submitted proposals-more than $50 \%$ of them had to be rejected for lack of beam time. Unfortunately, however, as many users of LANSCE have learned, the parasitic mode of facility operation, in which the nuclear physics program at LAMPF always had priority, has worked to our disadvantage - beam availability has been poor. And as support for nuclear physics has eroded over the past few years, run cycles have become shorter and shorter-this year we hope for a mere 1,000 hours of beam!

At a time when other parts of the neutron scattering infrastructure in the United States are suffering from the effects of long-term underinvestment, the closure of LANSCE is yet another blow to U.S. users. It implies that the United States will not participate significantly in mastering the technology of the present generation of high-intensity $(\approx 100 \mathrm{~kW})$ spallation neutron sources or in the exciting scientific research that such a source can support. So far, all the efforts to reverse the DOE closure decision have been in vain.

Los Alamos National Laboratory, of course, recognized the changing research paradigm described in the first paragraph and has made a proposal to convert the LAMPF complex into a dedicated spallation neutron facility with two targets and, in the initial stages, a beam

Material Matters is a forum for expressing personal points of view on issues of interest to the materials community. power of 1 megawatt. This proposal was one of those discussed at a recent meeting at Argonne National Laboratory, where the scientific case for a new spallation source was developed. While such a source would indeed be an exciting addition to the neutron scattering infrastructure, the laboratory has no illusions that the customary steps leading to such a construction project can be shortened. Even if the source were to be built at Los Alamos-and no such decision has yet been made-it would be many years before it would become available to the scientific community.

Los Alamos is, of course, better known for its design of nuclear weapons than it is for neutron scattering. In this area, too, enormous changes are occurring that we could only dream about and hope for a few years ago. In the new world, existing defense capabilities will be converted to civilian uses and the U.S. weapons laboratories-Los Alamos among them-are considering how the capabilities they have developed can be applied to civilian problems. This transformation of swords to ploughshares was discussed by President Clinton in a speech made during his May 17 visit to Los Alamos. One of the main civilian activities to which the Laboratory would like to apply its wealth of technical competence is research with neutron beams. Accordingly, the Laboratory is seeking some $\$ 50$ million in defense conversion funds for a short-term upgrade of LAMPF/ LANSCE that would provide an average proton current of $80+\mu \mathrm{A}$ at $20 \mathrm{~Hz}$ for neutron production. More importantly, from the users' perspective, the upgrade would allow reliable beam to be delivered for eight months or more each year and the facility would be dedicated to neutrons. The upgrade, which could be completed in less than two years-perhaps while LAMPF finished its most important nuclear physics experimentswould provide a much-needed research capability during the critical period before the Advanced Neutron Source at Oak Ridge National Laboratory becomes operational.

Roger Pynn is director of the Manuel Lujan Jr. Neutron Scattering Center at Los Alamos National Laboratory.

Editor's note: Funding for the continued operation and upgrade of LANSCE and for construction of the Advanced Neutron Source at Oak Ridge National Laboratory are subjects of ongoing congressional debate. 